\title{
The $K$-meson form factor and charge radius: linking low-energy data to future Jefferson Laboratory measurements
}

\author{
A. F. Krutov ${ }^{1, a}$, S. V. Troitsky ${ }^{2, b}$, V. E. Troitsky ${ }^{3, c}$ \\ ${ }^{1}$ Samara University, 443086 Samara, Russia \\ ${ }^{2}$ Institute for Nuclear Research of the Russian Academy of Sciences, 60th October Anniversary Prospect 7a, Moscow 117312, Russia \\ ${ }^{3}$ D.V. Skobeltsyn Institute of Nuclear Physics, M.V. Lomonosov Moscow State University, Moscow 119991, Russia
}

Received: 1 April 2017 / Accepted: 29 June 2017 / Published online: 12 July 2017

(C) The Author(s) 2017. This article is an open access publication

\begin{abstract}
Starting from a successful model of the $\pi$-meson electromagnetic form factor, we calculate a similar form factor, $F_{K}\left(Q^{2}\right)$, of the charged $K$ meson for a wide range of the momentum transfer squared, $Q^{2}$. The only remaining free parameter is to be determined from the measurements of the $K$-meson charge radius, $r_{K}$. We fit this single parameter to the published data of the NA-7 experiment which measured $F_{K}\left(Q^{2}\right)$ at $Q^{2} \rightarrow 0$ and determine our preferred range of $r_{K}$, which happens to be close to recent lattice results. Still, the accuracy in the determination of $r_{K}$ is poor. However, future measurements of the $K$-meson electromagnetic form factor at $Q^{2} \lesssim 5.5 \mathrm{GeV}^{2}$, scheduled in Jefferson Laboratory for 2017, will test our approach and will reduce the uncertainty in $r_{K}$ significantly.
\end{abstract}

\section{Introduction and outline}

Quantitative description of particle systems in the strongcoupling regime remains one of the most challenging problems of contemporary particle theory. The electromagnetic structure of light mesons represents an ideal testbed for various approaches to practical calculations at strong coupling, including low-energy effective theories of strong interactions and their connection to Quantum Chromodynamics (QCD). Not surprisingly, experimental understanding of various aspects of the meson structure comprises an important part of the scientific program of the upgraded Jefferson Laboratory (JLab) [1], presently ready for its start. In particular, the E12-09-011 experiment, scheduled in JLab Hall C for 2017, will attempt to measure the $K$-meson electromagnetic form factor, $F_{K}\left(Q^{2}\right)$, at the momentum transfer squared up

\footnotetext{
a e-mail: krutov@ssau.ru

b e-mail: st@ms2.inr.ac.ru

c e-mail: troitsky@theory.sinp.msu.ru
}

to $Q^{2} \sim 5.5 \mathrm{GeV}^{2}$ [2]. In this work, we address some important implications of this expected result and revisit previous scarce data on $F_{K}\left(Q^{2}\right)$ in the frameworks of a successful theoretical model.

Some time ago, a model for the electromagnetic form factor of the charged $\pi$ meson, $F_{\pi}\left(Q^{2}\right)$, has been developed (see Refs. [3-5] for a detailed description), possessing a few free parameters, fixed in 1998 [6] from the experimental data available at that time. The model predicted subsequent JLab experimental results on $F_{\pi}\left(Q^{2}\right)$ surprisingly well [7] without further tuning of parameters, despite the fact that the experimentally accessible range of $Q^{2}$ was extended by an order of magnitude [8]. Moreover, the model with the very same parameters predicts the correct QCD asymptotics of $F_{\pi}\left(Q^{2}\right)$ at large $Q^{2}[9,10]$. Subsequently, the model was also applied to the calculation of electroweak parameters of the $\rho$ meson [11], for which particular interesting relations have been obtained. The theoretical grounds for the model include a relativistic-invariant approximation [3] to the instant form of the relativistic Hamiltonian dynamics (see e.g. Ref. [12]), while the model's quantitative parameters in the light-quark sector are fixed from the successful $\pi$-meson study [6].

The two principal benefits of our model have been demonstrated in the $\pi$-meson study. The first one is its predictivity: provided the experimental value of the decay constant is fixed, only one parameter remains to fit the mean square radius. Any further dependence on the model details, e.g. in particular the choice of the phenomenological wave function, is negligible [6]. The second advantage is matching with QCD predictions in the ultraviolet limit: when constituentquark masses are switched off, as expected at high energies, the model reproduces correctly not only the functional form of the QCD asymptotics, but also the numerical coefficient; see Refs. $[9,10]$ for details. To the best of our knowledge, this is the only available low-energy model reproducing the QCD limit without introducing additional parameters. Analytical 
properties of the pion form factor, as a function on the complex $Q^{2}$ plane, obtained in our model, are the same as follows from the general quantum field theory principles [28]. Since the model predicted successfully the values of $F_{\pi}\left(Q^{2}\right)$ up to $Q^{2} \sim 2.5 \mathrm{GeV}^{2}$, we expect that it can be used for $F_{K}\left(Q^{2}\right)$ at least in the same domain of momentum transfers. Our model shares its limitations with other constituent-quark models of mesons: at present, their parameters cannot be consistently derived from QCD without additional experimental input.

In this paper, the model is applied to the $K$ meson. This brings two additional parameters into the game, one being the mass of the strange constituent quark, $M_{S}$, and another describing the interaction in the light-heavy quark system. In the case of the $\pi$ meson, the two corresponding parameters were uniquely determined from two measured observables, the $\pi$-meson decay constant, $f_{\pi}$, and charge radius, $r_{\pi}$, which determines the form-factor behavior at low $Q^{2}$. We will demonstrate below that, for the $K$ meson, the decay constant, $f_{K}$, fixes one combination of parameters, while the charge radius, $r_{K}$, is known with large uncertainties, which makes it difficult to use it for fixing the remaining parameter. We therefore keep it free and obtain a range of the model parameters consistent with the present data. We address old measurements of $F_{K}\left(Q^{2}\right)$ at $Q^{2} \rightarrow 0$ obtained in the NA7 experiment at CERN SPS [13], which represents the most precise source of experimental input for determination of $r_{K}$. The value of $r_{K}$ obtained in Ref. [13], since then extensively used in numerous experimental and theoretical works, was estimated from fitting the data points in the pole approximation. We demonstrate that the range of $Q^{2}$ studied in Ref. [13] was sufficiently large for deviations from this approximation to become important. We fit the NA-7 data points with our exact functions for $F_{K}\left(Q^{2}\right)$, which results in a slightly shifted value of $r_{K}$. We note that the obtained allowed range of $r_{K}$, bounded by the $68 \% \mathrm{CL}$ agreement with the data and by the consistency of the model, is in a better agreement with the recent lattice results [14] than the original result of Ref. [13].

Turning to higher energies, we use the constraints on $r_{K}$ to fix the allowed range of $F_{K}\left(Q^{2}\right)$ functions at modestly large $Q^{2} \lesssim 6 \mathrm{GeV}^{2}$. We observe that the spread of the theoretical curves exceeds considerably the expected precision of the E12-09-011 experiment in JLab. Therefore, within our approach, the E12-09-011 results might be used not only to study $F_{K}\left(Q^{2}\right)$ at moderate $Q^{2}$ but also to constrain its behavior at $Q^{2} \rightarrow 0$ and to further narrow the experimentally allowed range of $r_{K}$. This improvement in the value of a very soft parameter represents an unexpected application of the coming experiment, aimed presumably at the studies at much higher momentum transfers, $Q^{2} \sim(0.5-5.5) \mathrm{GeV}^{2}$.

The rest of the paper is organized as follows. In Sect. 2, we describe briefly the model for $F_{K}\left(Q^{2}\right)$, with the emphasis on the differences between the $\pi$ - and $K$-meson mod- els and on the two new parameters we have to introduce. Sect. 3 addresses the experimental constraints on $r_{K}$. The NA-7 data are reanalyzed here in the frameworks of our model. In Sect. 4, we present the expected $F_{K}\left(Q^{2}\right)$ behavior at larger $Q^{2}$ and demonstrate how the E12-09-011 JLab experiment may improve the precision of the $r_{K}$ measurement. We briefly conclude in Sect. 5 and present a more detailed description of the model in the Appendix.

\section{The $K$-meson electromagnetic form factor}

The approach we used is based on the instant-form Dirac relativistic Hamiltonian dynamics (see e.g. Ref. [12]) supplemented by the relativistic-invariant modified impulse approximation [3]. The form factor of a system of two quarks with different masses ${ }^{1}$ has been calculated, within this approach, in Ref. [15]. For completeness, all necessary formulas are collected in the Appendix.

In general, we need to know five parameters to proceed with the real numerical calculation. They include the masses of the two constituent quarks, $M_{u}$ and $M_{s}$; the parameter of the two-quark phenomenological wave function, $b$, with the physical meaning of the confinement scale; and two anomalous magnetic moments of quarks, $\kappa_{u}$ and $\kappa_{s}$. The values of the latter are fixed in the same way as it was done for the $\pi$ meson, that is through the Gerasimov sum rules; see Ref. [16] for details and the appendix for explicit expressions. The value of $\kappa_{u}$ is, clearly, the same as it was used for the $\pi$ meson. We also take advantage of the working model for $F_{\pi}\left(Q^{2}\right)$ where $M_{u}=0.22 \mathrm{GeV}$ was fixed. The phenomenological confinement scale, $b$, may in principle be different for different systems, and, for the moment, we keep it as a free parameter, together with $M_{s}$.

At this point, we note that we do not vary the shape of the phenomenological wave function $u(k)$ used in the calculation, but fix it instead from the $\pi$-meson study and leave only the scale $b$ as a free parameter. In early theoretical studies of our model $[15,18]$, various wave functions have been used, which, in general, resulted in different predictions. However, once the model was applied to phenomenology, the dependence on the wave-function choice was found negligible, provided the value of the meson decay constant was fixed [6]. The theoretical systematic uncertainty related to the choice of the wave-function shape is small compared to the experimental uncertainties. Note that the weak dependence of the results of calculation of electromagnetic form factors of pseudoscalar mesons on the shape of the wave function of constituent quarks has been pointed out also in Ref. [29].

\footnotetext{
${ }^{1}$ The $\pi$ meson is well described with $M_{u}=M_{d}$.
} 
The choice of the light constituent-quark mass $M_{u}$ was determined from a fit to experimental values of $F_{\pi}\left(Q^{2}\right)$ at small $Q^{2}$ in Ref. [6] and confirmed by subsequent experimental data. It is interesting to note that, with the same value of $M_{u}$, the mass spectrum of light mesons had been successfully described in Ref. [30], within a different framework. We leave the study of the meson masses in our model for future work.

Within our approach, we build up a consistent phenomenologically successful global fit of electroweak properties of light mesons. The same parameters have been used first for the best-studied $\pi$ meson $[6,10]$, then for certain properties of the $\rho$ meson [11]; now we proceed with the $K$ meson.

To fix the two free parameters, $b$ and $M_{u}$, for the $\pi$ meson, two experimental observables were used. One was the meson decay constant, $f_{\pi}$, and another was the meson charge radius, $r_{\pi}$, determined from experimental measurements of $F_{\pi}\left(Q^{2}\right)$ at $Q^{2} \rightarrow 0$. For the $K$ meson, we may equally well use the decay constant, $f_{K}=(0.1562 \pm 0.0010) \mathrm{GeV}$ [17], to eliminate one of the parameters. The expression relating $f_{K}$ to the model parameters was derived in Ref. [18] and is presented in the Appendix.

It would be natural to use the experimental information on the $K$-meson charge radius, $r_{K}$, to fix the single remaining free parameter and to predict the behavior of $F_{K}\left(Q^{2}\right)$ in the yet unexplored domain of large $Q^{2}$. However, as we will see in the next section, this approach is limited by the poor experimental knowledge of $r_{K}$.

\section{Experimental constraints on the $K$-meson charge radius}

The form factor $F_{K}\left(Q^{2}\right)$ was measured by the NA-7 experiment at CERN SPS, Ref. [13]. This measurement of 1986 remains the most recent and the most precise one, and we will concentrate on its results in what follows. ${ }^{2}$ Figure 1 presents the experimental data points. The authors of Ref. [13] used these data to extract the $K$-meson charge radius by fitting their data with the pole approximation,

$F_{K}\left(Q^{2}\right)=1 /\left(1+Q^{2}\left\langle r_{K}^{2}\right\rangle / 6\right)$.

We note in passing that a better fit to data points was obtained in Ref. [13] when the condition $F_{K}(0)=1$ was not used, though a departure from this condition is unphysical. This resulted in the value of $r_{K}^{2}=0.34 \pm 0.05 \mathrm{fm}^{2}(50 \% \mathrm{CL})$, widely used in subsequent studies.

However, one may note that the actual $F_{K}\left(Q^{2}\right)$ function may deviate from the pole approximation already at

2 Inclusion of an earlier measurement of Ref. [31] cannot change our result because of larger error bars and smaller number of data points, all of which lay farther away from $Q^{2}=0$.

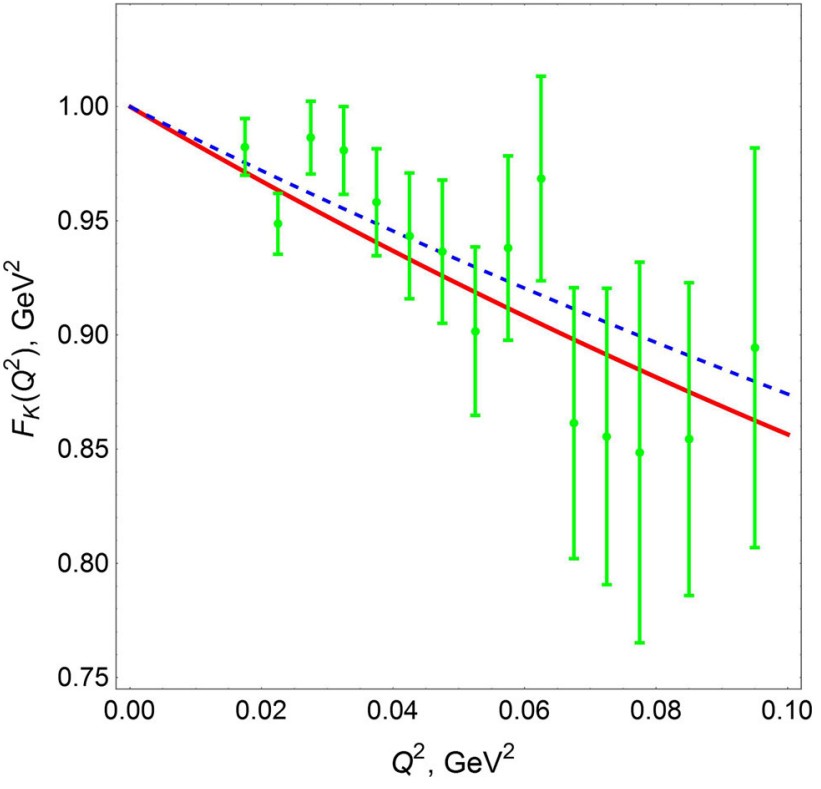

Fig. 1 The NA-7 measurement of $F_{K}\left(Q^{2}\right)$ at low $Q^{2}$. Data points from Ref. [13]. Blue dashed line the pole fit of Ref. [13]. Red full line the best-fit curve (this work)

$Q^{2} \sim 0.1 \mathrm{GeV}^{2}$, so that corrections to the pole approximation are already important for the NA-7 data range. This means that, to determine the derivative of $F_{K}\left(Q^{2}\right)$ at $Q^{2}=0$, and hence $r_{K}$, one should use either a shorter range of $Q^{2}$ or a more complicated approximation. The first option fails because of the insufficient number of data points. Fortunately, as described above, we can calculate the function $F_{K}\left(Q^{2}\right)$ within our approach. The model has one free parameter which we use to fit, by means of the usual $\chi^{2}$ method, the NA-7 data points with their experimental error bars. To do that, we consider the two-dimensional parameter space $\left(M_{s}, b\right)$ of the model; see Fig. 2.

Fixing the value of $f_{K}$ implies a constraint on $\left(M_{s}, b\right)$, so that a one-parametric space remains (one can see from Fig. 2 that the precision of the experimental value of $f_{K}$ is so good that its uncertainty may be neglected). The remaining freedom is therefore parametrized by a line on the $\left(M_{s}, b\right)$ plane; different points on the line correspond to different values of $r_{K}$. Changing $M_{S}$ and always keeping $b\left(M_{S}\right)$ to satisfy the $f_{K}$ constraint, one may fit the experimental data points for $F_{K}\left(Q^{2}\right)$. One may note, however, from Fig. 2, that not all values of $r_{K}$ may be achieved, provided the $f_{K}$ constraint is satisfied. Indeed, at $\left\langle r_{K}^{2}\left(M_{s}, b\right)\right\rangle<0.39 \mathrm{fm}^{2}$, the two curves determined by $f_{K}\left(M_{s}, b\right)$ and $r_{K}\left(M_{s}, b\right)$, Fig. 2, have no intersection points. Since all other parameters beyond $M_{S}$ and $b$ are fixed from the $\pi$-meson studies and their values are confirmed experimentally, we have no freedom to change this picture. Therefore, the limitation in simultaneous description of $f_{K}$ and $r_{K}$ is a consequence of our requirement of a joint description of $\pi$ and $K$ mesons, and not of the construction 


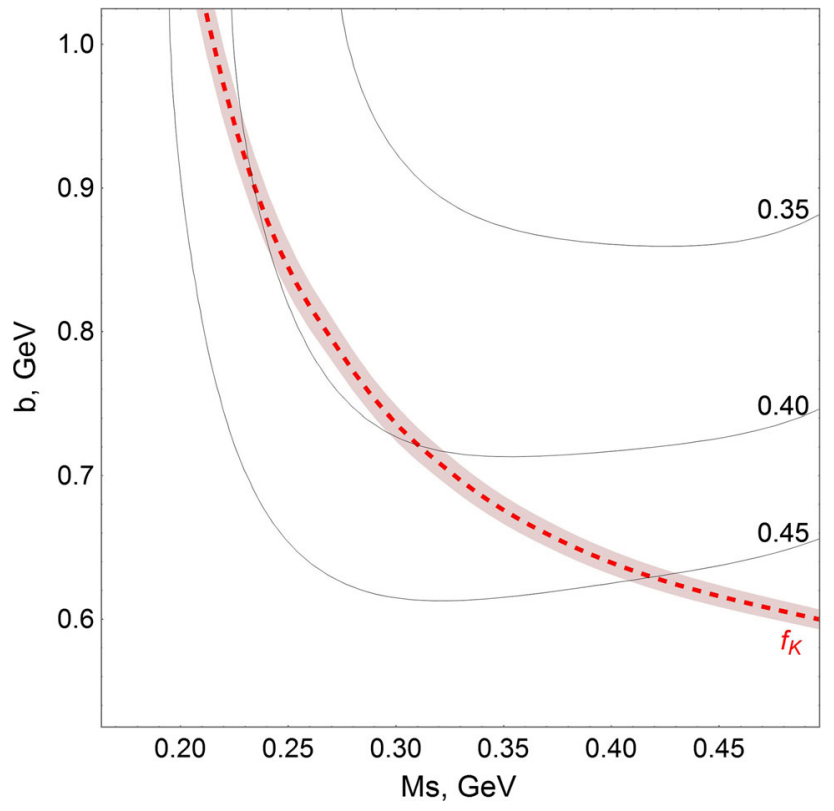

Fig. 2 The two parameters for the $K$-meson form factor. The experimental value of $f_{K}$ is reproduced on the red dashed line, with the pink shade representing the experimental uncertainty (this condition leaves essentially one-dimensional parameter space). Thin black lines correspond to different values of $r_{K}^{2}$, indicated by numbers (in $\mathrm{fm}^{2}$ )

of our relativistic model, whose predictivity is manifested in this way. The value of $M_{s} \approx 0.27 \mathrm{GeV}$ corresponds to the lowest achievable $r_{K}$, which splits the $b\left(M_{s}\right)$ line into two branches, so that a larger value of $r_{K}$ may be obtained for two distinct values of $M_{s}$.

In Fig. 3, we illustrate the results of the fit by presenting the $\chi^{2}\left(r_{K}\right)$ function determined by this method. We note that the best fit $\left(\chi^{2}=13.5\right.$ for 14 degrees of freedom) corresponds to the lowest value of $r_{K}$, allowed in our approach, and that

$0.39 \mathrm{fm}^{2} \leq\left\langle r_{K}^{2}\right\rangle \leq 0.42 \mathrm{fm}^{2}(68 \% \mathrm{CL}$, this work $)$.

Our best-fit curve is also presented in Fig. 1. It is interesting to note that our best-fit value of $\left\langle r_{K}^{2}\right\rangle=0.39 \mathrm{fm}^{2}$ is in a better agreement with the recent lattice calculations [14], $\left\langle r_{K \text {, lattice }}^{2}\right\rangle=0.380 \pm 0.033 \mathrm{fm}^{2}$, than the best-fit value derived with the pole approximation. However, we note that, being derived from the same data, our value for $r_{K}$ shares similar large statistical uncertainties with the original result, and both are compatible at $68 \% \mathrm{CL}$. It is interesting to compare our result also with the values obtained from the data analysis in the frameworks of the chiral perturbation theory [32], which vary between $\left\langle r_{K, \mathrm{ChPT} \text {,min }}^{2}\right\rangle=0.354 \pm 0.071 \mathrm{fm}^{2}$ and $\left\langle r_{K, \mathrm{ChPT}, \max }^{2}\right\rangle=0.431 \pm 0.071 \mathrm{fm}^{2}$ : our $68 \%$ CL interval for $\left\langle r_{K}^{2}\right\rangle$ is contained in that of Ref. [32] for all their assumptions.

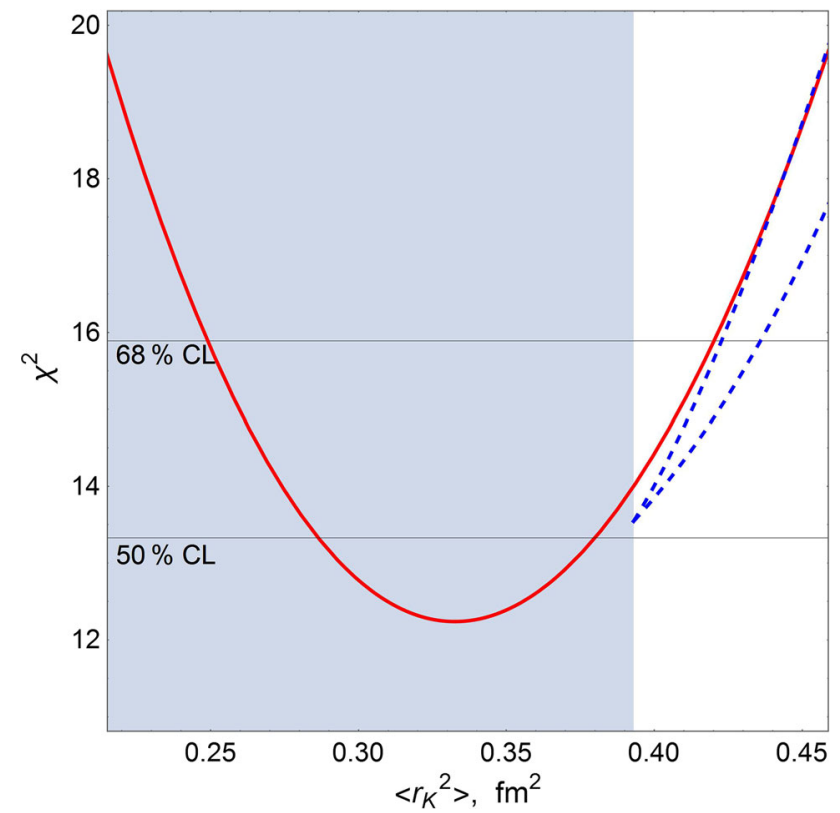

Fig. 3 The $\chi^{2}\left(r_{K}\right)$ function for our fits (blue dashed lines for two branches; see text). The shaded range, $r_{K}^{2}<0.39 \mathrm{fm}^{2}$, is not compatible with our model. For comparison, the $\chi^{2}\left(r_{K}\right)$ function for the pole fit of Ref. [13] is shown by the full red line. Horizontal lines bound the $50 \%$ CL (used in Ref. [13]) and 68\% CL ranges

\section{Large $Q^{2}$ and the future JLab experiment}

The use of the low- $Q^{2}$ data constrains, by Eq. (1), the remaining free parameter of the model through the fitting procedure described in the previous section. In principle, this allows us to calculate the $F_{K}\left(Q^{2}\right)$ function for a large range of $Q^{2}$ and to make predictions for the future JLab measurements. This prediction is presented in Fig. 4, where the $Q^{2}$ range probed by the E12-09-011 JLab experiment and estimated uncertainties of the measurement [27] are shown. For comparison, we present also predictions obtained within other approaches.

Considering Fig. 4, one immediately notes that the uncertainty in our predictions, determined by the uncertainty in the $r_{K}$ measurements, exceeds the expected precision of the experiment and is of order the typical difference between model predictions. The E12-09-011 experiment would be able to distinguish between our model and several other ones.

Since these approaches differ in their original assumptions as well as in approximations being used, future JLab experiments will be able, in principle, to contribute to the choice between various models of the description of nonperturbative dynamics of strong interactions at large and intermediate distances. In this context, it is instructive to compare our results with those of Ref. [24]. Their approach differs from ours by the choice of the form of relativistic dynamics: they use the light-front dynamics. Another difference is in the approximations: while we use the so-called modified 


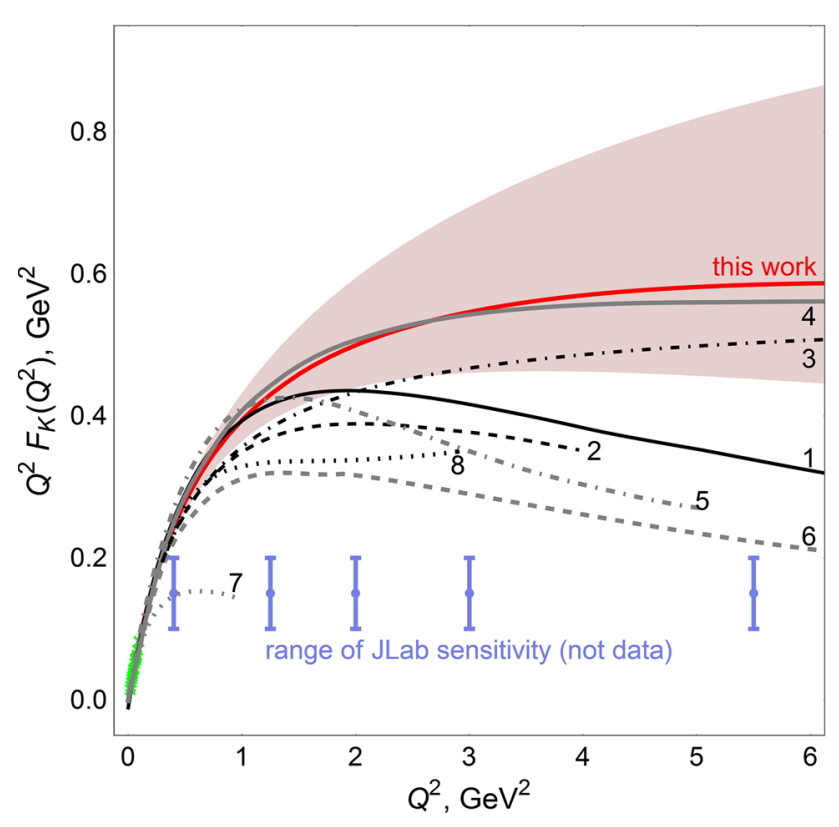

Fig. 4 Predictions of our model for $F_{K}\left(Q^{2}\right)$ are parametrized by a single free parameter which we fit to the low-energy data of Ref. [13] (green crosses at $Q^{2} \rightarrow 0$ ). The red line represents the best fit and the pink shaded area represents the $68 \%$ CL range. Also shown are predictions obtained in other works. 1 (full black): Ref. [19]; 2 (dashed black): Ref. [20]; 3 (dot-dashed black): Ref. [21]; 4 (full gray): Ref. [22]; 5 (dot-dashed gray): Ref. [23]; 6 (dashed gray): Ref. [24]; 7 (dotted gray): Ref. [25]; 8 (dotted black): Ref. [26]. Points with error bars are not data points, they represent the $Q^{2}$ range and projected uncertainties of the E12-09-011 JLab experiment [27]

impulse approximation, see e.g. Ref. [5], the conventional impulse approximation was used in Ref. [24]. Our modified impulse approximation, unlike the conventional one, does not violate covariance conditions, nor the current conservation. Another difference with Ref. [24], which may be important at large $Q^{2}$, is in the $Q^{2}$-dependence of quark form factors: we use a renormalization-group inspired logarithmic function, while in Ref. [24], a dipole form is used. Note that the value of $M_{u}$ and the expression for the quark radius coincide in the two works.

In addition, this consideration opens a surprising new possibility to constrain the low- $Q^{2}$ behavior of $F_{K}\left(Q^{2}\right)$ and to reduce the experimental uncertainty in $r_{K}$. Indeed, the expected error bars of the experiment are smaller than the spread of $F_{K}\left(Q^{2}\right)$ curves predicted in our model. This spread is determined by the $68 \%$ C.L. allowed variations of the single not firmly fixed parameter of the model, $r_{K}$, which determines the behavior of $F_{K}\left(Q^{2}\right)$ at $Q^{2} \rightarrow 0$. Hence, limiting the spread at large $Q^{2}$ would narrow the allowed range of $r_{K}$. Figure 5 illustrates how the measurement of $F_{K}\left(5.5 \mathrm{GeV}^{2}\right)$ in JLab would constrain $r_{K}$ in the case that the uncertainty of the measurement agrees with the estimate of Ref. [27]. We note that the reduced uncertainty in $r_{K}$ transforms, within our approach, into a more precise knowledge of the model

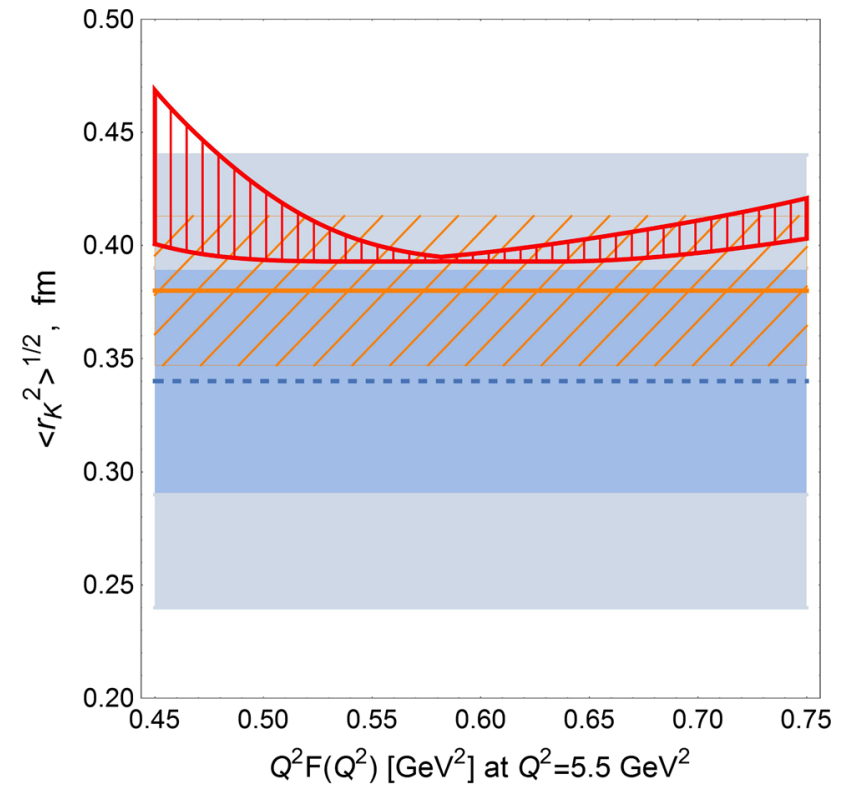

Fig. 5 The effect of future JLab measurements on the precision of the $r_{K}$ value. Suppose that JLab finds the value of $F_{K}\left(Q^{2}=5.5 \mathrm{GeV}^{2}\right)$ shown in the horizontal axis, with the uncertainty given in Ref. [27] (conservative value). Then, within our approach, one would be able to constrain $r_{K}$ to lay between the lower and upper thick red curves for this value of $F_{K}$ (the strip with red vertical hatching). For comparison, $r_{K}$ of Ref. [13] is shown by the dashed line (best fit), dark blue shade (68\% CL) and light blue shade (95\% CL). The horizontal strip with orange diagonal hatching represents the lattice result of Ref. [14]

parameter; for instance, $M_{s}$, which may be interesting from a theoretical point of view (cf. Fig. 2).

\section{Conclusions}

In this work, we discussed present and future experimental data on the $K$-meson electromagnetic form factor, $F_{K}\left(Q^{2}\right)$, in the context of the model for the electroweak structure of light mesons based on the relativistic-invariant modified impulse approximation in the frameworks of the instant-form relativistic Hamiltonian dynamics. All but two parameters of the model are fixed from its successful application to the $\pi$-meson form factor in previous studies, where, experimentally, the model predictions had been confirmed precisely by a number of subsequent measurements, and theoretically, the correct QCD asymptotics was reproduced. Of the two parameters specific for the $K$-meson case, one combination is fixed from the $K$-meson decay constant, $f_{K}$, while the remaining one is related to the $K$-meson charge radius, $r_{K}$. The latter is known experimentally with large uncertainties. We revisited the NA-7 data on $F_{K}\left(Q^{2}\right)$ at $Q^{2} \lesssim 0.1 \mathrm{GeV}^{2}$ and found the $r_{K}$ range allowed by the data within our model. This range agrees well with the recent lattice results. Still, the error bars in $r_{K}$ remain large, which makes the predictions 
of the model uncertain in the large- $Q^{2}$ range to be probed by the coming E12-09-011 experiment in JLab. However, this suggested an unexpected application of the coming JLab measurements of $F_{K}\left(Q^{2}\right)$, which, despite being performed at $Q^{2} \sim(0.5-5) \mathrm{GeV}^{2}$, would improve the accuracy of the $r_{K}$ measurements.

Acknowledgements We thank A. Gasparian and G. Huber for interesting information on the coming JLab $K$-meson experiments. The work of AK was supported in part by the Ministry of Education and Science of the Russian Federation (Grant No. 1394, state task). The work of ST on the nonperturbative description of strongly coupled QCD bound states is supported by the Russian Science Foundation, Grant 14-22-00161.

Open Access This article is distributed under the terms of the Creative Commons Attribution 4.0 International License (http://creativecomm ons.org/licenses/by/4.0/), which permits unrestricted use, distribution, and reproduction in any medium, provided you give appropriate credit to the original author(s) and the source, provide a link to the Creative Commons license, and indicate if changes were made.

Funded by SCOAP ${ }^{3}$.

\section{Appendix A: Formulas for the form-factor calculation}

Appendix A.1: The form factor of a system with two quarks of different masses

This form factor was calculated, in the present approach, in Ref. [15].

The free form factor is given by

$$
\begin{aligned}
g_{0} & \left(s, Q^{2}, s^{\prime}\right) \\
= & \frac{\sqrt{s s^{\prime}}}{\sqrt{\left[s^{2}-2 s\left(M_{s}^{2}+M_{u}^{2}\right)+\eta^{2}\right]\left[s^{\prime 2}-2 s^{\prime}\left(M_{s}^{2}+M_{u}^{2}\right)+\eta^{2}\right]}} \\
& \times \frac{Q^{2}\left(s+s^{\prime}+Q^{2}\right)}{2\left[\lambda\left(s,-Q^{2}, s^{\prime}\right)\right]^{3 / 2}}\left(B^{u}\left(s, Q^{2}, s^{\prime}\right)+B^{\bar{s}}\left(s, Q^{2}, s^{\prime}\right)\right),
\end{aligned}
$$

where $M_{q}$ is the mass of the constituent quark $q, \eta=M_{u}^{2}-$ $M_{s}^{2}$,

$$
\begin{aligned}
& B^{\bar{s}}\left(s, Q^{2}, s^{\prime}\right)=\left[f_{1}^{(\bar{s})}\left(s+s^{\prime}+Q^{2}-2 \eta\right) \cos \left(\omega_{1}+\omega_{2}\right)\right. \\
& \left.\quad-f_{2}^{(\bar{s})} \frac{M_{s}}{2} \xi\left(s, Q^{2}, s^{\prime}\right) \sin \left(\omega_{1}+\omega_{2}\right)\right] \theta\left(s, Q^{2}, s^{\prime}\right), \\
& \xi\left(s, Q^{2}, s^{\prime}\right) \\
& =\sqrt{-\lambda\left(s,-Q^{2}, s^{\prime}\right) M_{s}^{2}+s s^{\prime} Q^{2}-\eta Q^{2}\left(s+s^{\prime}+Q^{2}\right)+Q^{2} \eta^{2},}
\end{aligned}
$$

$$
\lambda(a, b, c)=a^{2}+b^{2}+c^{2}-2(a b+a c+b c),
$$$$
f_{1}^{(\bar{s})}=\frac{2 M_{s} G_{E}^{(\bar{s})}\left(Q^{2}\right)}{\sqrt{4 M_{s}^{2}+Q^{2}}} ;
$$$$
f_{2}^{(\bar{s})}=-\frac{4 G_{M}^{(\bar{s})}\left(Q^{2}\right)}{M_{s} \sqrt{4 M_{s}^{2}+Q^{2}}},
$$

the Wigner rotation parameters are

$$
\begin{aligned}
\omega_{1}= & \arctan \frac{\xi\left(s, Q^{2}, s^{\prime}\right)}{M_{u}\left[\left(\sqrt{s}+\sqrt{s^{\prime}}\right)^{2}+Q^{2}\right]+\left(\sqrt{s}+\sqrt{s^{\prime}}\right)\left(\sqrt{s s^{\prime}}+\eta\right)}, \\
\omega_{2}= & \arctan \left[\left(\sqrt{s}+\sqrt{s^{\prime}}+2 M_{s}\right) \xi\left(s, Q^{2}, s^{\prime}\right)\right. \\
& \times\left\{M_{s}\left(s+s^{\prime}+Q^{2}\right)\left(\sqrt{s}+\sqrt{s^{\prime}}+2 M_{s}\right)\right. \\
& \left.\left.+\sqrt{s s^{\prime}}\left(4 M_{s}^{2}+Q^{2}\right)-\eta\left[2 M_{s}\left(\sqrt{s}+\sqrt{s^{\prime}}\right)-Q^{2}\right]\right\}^{-1}\right],
\end{aligned}
$$

$\theta\left(s, Q^{2}, s^{\prime}\right)=\vartheta\left(s^{\prime}-s_{1}\right)-\vartheta\left(s^{\prime}-s_{2}\right)$,

$\vartheta$ is the conventional step function,

$$
\begin{aligned}
s_{1,2}= & M_{s}^{2}+M_{u}^{2}+\frac{1}{2 M_{s}^{2}}\left(2 M_{s}^{2}+Q^{2}\right)\left(s-M_{s}^{2}-M_{u}^{2}\right) \\
& \mp \frac{1}{2 M_{s}^{2}} \sqrt{Q^{2}\left(4 M_{s}^{2}+Q^{2}\right)\left[s^{2}-2 s\left(M_{s}^{2}+M_{u}^{2}\right)+\eta^{2}\right]} .
\end{aligned}
$$

The function $B^{u}\left(s, Q^{2}, s^{\prime}\right)$ is obtained from $B^{\bar{s}}\left(s, Q^{2}, s^{\prime}\right)$ by the substitution $M_{s} \leftrightarrow M_{u}$ everywhere.

The quark form factors are

$$
G_{E}^{q}\left(Q^{2}\right)=e_{q} f_{q}\left(Q^{2}\right), \quad G_{M}^{q}\left(Q^{2}\right)=\left(e_{q}+\kappa_{q}\right) f_{q}\left(Q^{2}\right),
$$

where $e_{q}$ are quark charges, $f_{q}\left(Q^{2}\right)=1 /\left(1+\log \left(r_{q}^{2} Q^{2} / 6\right)\right)$ and $r_{q}^{2}=0.3 / M_{q}^{2}$.

The anomalous magnetic moments $\kappa_{q}$ of quarks $q$ are calculated following Ref. [16]. The values of $\kappa_{u}$ and $\kappa_{\bar{d}}$ should satisfy [16]

$\frac{e_{u}+\kappa_{u}}{e_{d}+\kappa_{d}}=-1.77$

The $\pi$-meson form factor depends [6] on the sum $\kappa_{u}+\kappa_{\bar{d}}$, which has been fixed in Ref. [6] from the condition that the constituent-quark parameters providing a good description of the data do not depend on the choice of the shape of the phenomenological wave function: in this way, the value $\kappa_{u}+$ $\kappa_{\bar{d}}=0.0268$ has been found for $M_{u}=0.22 \mathrm{GeV}$, and we use this value in the present study. Together with Eq. (A.1), this condition determines $\kappa_{u}$ and $\kappa_{\bar{d}}$ unambiguously. The value of $\kappa_{s}$ is determined [16] from

$$
\left(\frac{\frac{e_{u}+\kappa_{u}}{e_{d}+\kappa_{d}}+\frac{e_{s}+\kappa_{s}}{e_{d}+\kappa_{d}}}{1+\frac{e_{s}+\kappa_{s}}{e_{d}+\kappa_{d}}}\right)^{2}=0.42 .
$$

This relation, for fixed $\kappa_{u}$ and $\kappa_{\bar{d}}$, determines the value of $\kappa_{\bar{S}}$ up to the choice of the sign at the square root from the right-hand side. We choose the negative sign because in the opposite case, the solution gives an unphysically large value of $\kappa_{\bar{S}}$. We arrive at the values of $\kappa_{u} \approx-0.01055$ and $\kappa_{\bar{s}} \approx$ -0.08099 , which are used in this study.

The form factor $F_{K}\left(Q^{2}\right)$ is given by the double integral,

$$
F_{K}\left(Q^{2}\right)=\int \mathrm{d} \sqrt{s} \mathrm{~d} \sqrt{s^{\prime}} \varphi(k) g_{0}\left(s, Q^{2}, s^{\prime}\right) \varphi\left(k^{\prime}\right),
$$


where

$\varphi(k)=\sqrt{\sqrt{s}\left(1-\eta^{2} / s^{2}\right)} u(k) k$,

$k=\sqrt{\left(s^{2}-2 s\left(M_{s}^{2}+M_{u}^{2}\right)+\eta^{2}\right) / 4 s}$,

is the phenomenological wave function of the two-quark system. Following previous studies of the $\pi$ meson, we choose the power-law function,

$u(k)=N\left(k^{2} / b^{2}+1\right)^{-3}$,

where the normalization $N=16 \sqrt{2 /\left(7 \pi b^{3}\right)}$ is determined by the condition

$\int_{0}^{\infty} \mathrm{d} k k^{2} u(k)^{2}=1$.

Appendix A.2: The $K$-meson decay constant

The expression for the decay constant $f_{K}$ was calculated, in the present approach, in Ref. [18]. It reads

$f_{K}=\int_{M_{s}+M_{u}}^{\infty} \mathrm{d} \sqrt{s} G_{0}(s) \varphi(s)$,

where

$$
\begin{aligned}
G_{0}(s)= & \frac{\sqrt{3}}{2 \sqrt{2} \pi \sqrt{s}} \sqrt{\left(p_{s 0}+M_{s}\right)\left(p_{u 0}+M_{u}\right)} \\
& \times\left(1-\frac{k^{2}}{\left(p_{s 0}+M_{s}\right)\left(p_{u 0}+M_{u}\right)}\right), \\
p_{q 0}= & \sqrt{M_{q}^{2}+k^{2}} .
\end{aligned}
$$

\section{References}

1. J. Dudek et al., Physics opportunities with the $12 \mathrm{GeV}$ upgrade at Jefferson Lab. Eur. Phys. J. A 48, 187 (2012). arXiv:1208.1244 [hep-ex]

2. P. Bosted et al., Studies of the L-T separated kaon electroproduction cross section from 5-11 GeV. Proposal PR12-09-011 to Jefferson Lab PAC 34 (2008). Approved as E12-09-011. https://www.jlab. org/exp_prog/generated/12GeV/apphallc.html

3. A.F. Krutov, V.E. Troitsky, Relativistic instant form approach to the structure of two-body composite systems. Phys. Rev. C 65, 045501 (2002). arXiv:hep-ph/0204053

4. A.F. Krutov, V.E. Troitsky, Relativistic instant form approach to the structure of two-body composite systems. 2. Nonzero spin. Phys. Rev. C 68, 018501 (2003). arXiv:hep-ph/0210046

5. A.F. Krutov, V.E. Troitsky, Instant form of Poincare-invariant quantum mechanics and description of the structure of composite systems. Phys. Part. Nucl. 40, 136 (2009)

6. A.F. Krutov, V.E. Troitsky, On a possible estimation of the constituent quark parameters from Jefferson Lab experiments on pion form-factor. Eur. Phys. J. C 20, 71 (2001). arXiv:hep-ph/9811318

7. A.F. Krutov, V.E. Troitsky, N.A. Tsirova, Nonperturbative relativistic approach to pion form factor versus JLab experiments. Phys. Rev. C 80, 055210 (2009). arXiv:0910.3604 [nucl-th]
8. G.M. Huber et al., [Jefferson Lab Collaboration], Charged pion form-factor between $Q^{2}=0.60 \mathrm{GeV}^{2}$ and $2.45 \mathrm{GeV}^{2}$. II. Determination of, and results for, the pion form-factor. Phys. Rev. C 78, 045203 (2008). arXiv:0809.3052 [nucl-ex]

9. A.F. Krutov and V.E. Troitsky, Asymptotic estimates of the pion charge form-factor. Theor. Math. Phys. 116, 907 (1998) [Teor. Mat. Fiz. 116 (1998) 215]

10. S.V. Troitsky, V.E. Troitsky, Transition from a relativistic constituent-quark model to the quantum-chromodynamical asymptotics: a quantitative description of the pion electromagnetic form factor at intermediate values of the momentum transfer. Phys. Rev. D 88, 093005 (2013). arXiv:1310.1770 [hep-ph]

11. A.F. Krutov, R.G. Polezhaev, V.E. Troitsky, The radius of the $\rho$ meson determined from its decay constant. Phys. Rev. D 93, 036007 (2016). arXiv:1602.00907 [hep-ph]

12. B.D. Keister, W.N. Polyzou, Relativistic Hamiltonian dynamics in nuclear and particle physics. Adv. Nucl. Phys. 20, 225 (1991)

13. S.R. Amendolia et al., A measurement of the kaon charge radius. Phys. Lett. B 178, 435 (1986)

14. S. Aoki et al., [JLQCD Collaboration], Light meson electromagnetic form factors from three-flavor lattice QCD with exact chiral symmetry. Phys. Rev. D 93, 034504 (2016). arXiv:1510.06470 [hep-lat]

15. E.V. Balandina, A.F. Krutov, V.E. Troitsky, Elastic charge formfactors of $\pi$ and $K$ mesons. J. Phys. G 22, 1585 (1996). arXiv:hep-ph/9508248

16. S.B. Gerasimov, Electroweak moments of baryons and hidden strangeness of the nucleon. Chin. J. Phys. 34, 848 (1996). arXiv:hep-ph/9906386

17. K.A. Olive et al., [Particle Data Group], Review of particle physics. Chin. Phys. C 38, 090001 (2014)

18. A.F. Krutov, Electroweak properties of light mesons in the relativistic model of constituent quarks. Phys. Atom. Nucl. 60, 1305 (1997) [Yad. Fiz. 60 (1997) 1442]

19. J. He, B. Julia-Diaz, Y.B. Dong, Electroweak properties of the $\pi$, $K$ and $K^{*}(892)$ in the three forms of relativistic kinematics. Eur. Phys. J. A 24, 411 (2005). arXiv:hep-ph/0503294

20. P. Maris, P.C. Tandy, The $\pi, K^{+}$, and $K^{0}$ electromagnetic formfactors. Phys. Rev. C 62, 055204 (2000). arXiv:nucl-th/0005015

21. Y. Ninomiya, W. Bentz, I.C. Cloet, Dressed quark mass dependence of pion and kaon form factors. Phys. Rev. C 91, 025202 (2015). arXiv:1406.7212 [nucl-th]

22. P.T.P. Hutauruk, I.C. Cloet, A.W. Thomas, Flavor dependence of the pion and kaon form factors and parton distribution functions. Phys. Rev. C 94, 035201 (2016). arXiv:1604.02853 [nucl-th]

23. C.J. Burden, C.D. Roberts, M.J. Thomson, Electromagnetic formfactors of charged and neutral kaons. Phys. Lett. B 371, 163 (1996). arXiv:nucl-th/9511012

24. F. Cardarelli, I.L. Grach, I.M. Narodetsky, E. Pace, G. Salme, S. Simula, Charge form-factor of pi and K mesons. Phys. Rev. D 53, 6682 (1996). arXiv:nucl-th/9507038

25. E.O. da Silva, J.P.B.C. de Melo, B. El-Bennich, V.S. Filho, Pion and kaon elastic form factors in a refined light-front model. Phys. Rev. C 86, 038202 (2012). arXiv:1206.4721 [nucl-th]

26. D. Ebert, R.N. Faustov, V.O. Galkin, Masses and electroweak properties of light mesons in the relativistic quark model. Eur. Phys. J. C 47, 745 (2006). arXiv:hep-ph/0511029

27. T. Horn, C.D. Roberts, The pion: an enigma within the standard model. J. Phys. G 43, 073001 (2016). arXiv:1602.04016 [nucl-th]

28. A.F. Krutov, M.A. Nefedov, V.E. Troitsky, Analytic continuation of the pion form factor from the spacelike to the timelike domain. Theor. Math. Phys. 174, 331 (2013)

29. F. Schlumpf, Charge form-factors of pseudoscalar mesons. Phys. Rev. D 50, 6895 (1994). doi:10.1103/PhysRevD.50.6895. arXiv:hep-ph/9406267 
30. S. Godfrey, N. Isgur, Mesons in a relativized quark model with chromodynamics. Phys. Rev. D 32, 189 (1985)

31. E.B. Dally et al., Direct measurement of the negative kaon formfactor. Phys. Rev. Lett. 45, 232 (1980)
32. J. Bijnens, P. Talavera, Pion and kaon electromagnetic form-factors. JHEP 0203, 046 (2002). arXiv:hep-ph/0203049 